CUBO A Mathematical Journal

Vol.12, $N^{\underline{O}} 01$, (95-102). March 2010

\title{
An Identity Related to Derivations of Standard Operator Algebras and Semisimple $\mathrm{H}^{*}$-Algebras ${ }^{1}$
}

\author{
IRENA KOSI-ULBL \\ Faculty of Mechanical Engineering, University of Maribor, \\ Smetanova 17, Maribor, Slovenia \\ email: irena.kosi@uni-mb.si \\ AND \\ JOSO VUKMAN \\ Department of Mathematics and Computer Science, Faculty of Natural Sciences and \\ Mathematics, \\ University of Maribor, Koroška 160, Maribor, Slovenia \\ email: joso.vukman@uni-mb.si
}

\begin{abstract}
In this paper we prove the following result. Let $X$ be a real or complex Banach space, let $L(X)$ be the algebra of all bounded linear operators on $X$, and let $A(X) \subset L(X)$ be a standard operator algebra. Suppose $D: A(X) \rightarrow L(X)$ is a linear mapping satisfying the relation $D\left(A^{n}\right)=\sum_{j=1}^{n} A^{n-j} D(A) A^{j-1}$ for all $A \in A(X)$. In this case $D$ is of the form $D(A)=A B-B A$, for all $A \in A(X)$ and some $B \in L(X)$, which means that $D$ is a linear derivation. In particular, $D$ is continuous. We apply this result, which generalizes a classical result of Chernoff, to semisimple $H^{*}$-algebras.

This research has been motivated by the work of Herstein [4], Chernoff [2] and Molnár [5] and is a continuation of our recent work [8] and [9]. Throughout, $R$ will represent an associative ring. Given an integer $n \geq 2$, a ring $R$ is said to be $n$-torsion free, if for $x \in R, n x=0$
\end{abstract}

\footnotetext{
${ }^{1}$ This research has been supported by the Research Council of Slovenia
} 
implies $x=0$. Recall that a ring $R$ is prime if for $a, b \in R, a R b=(0)$ implies that either $a=0$ or $b=0$, and is semiprime in case $a R a=(0)$ implies $a=0$. Let $A$ be an algebra over the real or complex field and let $B$ be a subalgebra of $A$. A linear mapping $D: B \rightarrow A$ is called a linear derivation in case $D(x y)=D(x) y+x D(y)$ holds for all pairs $x, y \in R$. In case we have a ring $R$ an additive mapping $D: R \rightarrow R$ is called a derivation if $D(x y)=D(x) y+x D(y)$ holds for all pairs $x, y \in R$ and is called a Jordan derivation in case $D\left(x^{2}\right)=D(x) x+x D(x)$ is fulfilled for all $x \in R$. A derivation $D$ is inner in case there exists $a \in R$, such that $D(x)=a x-x a$ holds for all $x \in R$. Every derivation is a Jordan derivation. The converse is in general not true. A classical result of Herstein [4] asserts that any Jordan derivation on a prime ring of characteristic different from two is a derivation. Cusack [3] generalized Herstein's result to 2-torsion free semiprime rings. Let us recall that a semisimple $H^{*}$-algebra is a semisimple Banach ${ }^{*}$-algebra whose norm is a Hilbert space norm such that $\left(x, y z^{*}\right)=(x z, y)=\left(z, x^{*} y\right)$ is fulfilled for all $x, y, z \in A$ (see [1]). Let $X$ be a real or complex Banach space and let $L(X)$ and $F(X)$ denote the algebra of all bounded linear operators on $X$ and the ideal of all finite rank operators in $L(X)$, respectively. An algebra $A(X) \subset L(X)$ is said to be standard in case $F(X) \subset A(X)$. Let us point out that any standard algebra is prime, which is a consequence of Hahn-Banach theorem.

\section{RESUMEN}

En este artículo nosotros provamos el seguiente resultado. Sea $X$ un espacio de Banach real o complejo, sea $L(X)$ a algebra de todos los operadores linares acotados sobre $X, y$ sea $A(X) \subset L(X)$ una algebra de operadores estandar. Suponga $D: A(X) \longrightarrow L(X)$ una aplicación lineal verificando la relación $D\left(A^{n}\right)=\sum_{j=1}^{n} A^{n-j} D(A) A^{j-1}$ para todo $A \in A(X)$. En este caso $D$ es de la forma $D(A)=A B-B A$, para todo $A \in A(X)$ y algún $B \in L(X)$, lo que significa que $D$ es una deriviación lineal. En particual, $D$ es continua. Nosotros aplicamos este resultado el cual generaliza un resultado clásico de Chernoff, para $H^{*}$-algebras semisimple. Este trabajo fué motivado por un trabajo de Herstein [4], Chernoff [2] y Molnár [5] y este una continuación de nuestro reciente trabajo [8] y [9].

Key words and phrases: Prime ring, semiprime ring, Banach space, standard operator algebra, $H^{*}$-algebra, derivation, Jordan derivation.

Math. Subj. Class.: 46K15, 46H99, $13 N 15$.

Let us start with the following result proved by Chernoff [2] (see also [6] and [8]).

THEOREM A. Let $X$ be a real or complex Banach space and let $A(X)$ be a standard operator algebra on $X$. Let $D: A(X) \rightarrow L(X)$ be a linear derivation. In this case $D$ is of the form $D(A)=A B-B A$, for all $A \in A(X)$ and some $B \in L(X)$. In particular, $D$ is continuous.

It is our aim in this paper to prove the following result which generalizes Theorem $\mathrm{A}$.

THEOREM 1. Let $X$ be a real or complex Banach space and let $A(X)$ be a standard operator 
algebra on $X$. Suppose $D: A(X) \rightarrow L(X)$ is a linear mapping satisfying the relation

$$
D\left(A^{n}\right)=\sum_{j=1}^{n} A^{n-j} D(A) A^{j-1} .
$$

for all $A \in A(X)$. In this case $D$ is of the form $D(A)=A B-B A$, for all $A \in A(X)$ and some $B \in L(X)$, which means that $D$ is a linear derivation. In particular, $D$ is continuous.

Proof. We

have the relation

$$
D\left(A^{n}\right)=\sum_{j=1}^{n} A^{n-j} D(A) A^{j-1}
$$

Let $A$ be from $F(X)$ and let $P \in F(X)$, be a projection with $A P=P A=A$. From the above relation one obtains

$$
D(P)=P D(P)+(n-2) P D(P) P+D(P) P .
$$

Right multiplication of the relation (2) by $P$ gives

$$
P D(P) P=0 .
$$

Putting $A+P$ for $A$ in the relation (1), we obtain

$$
\begin{aligned}
& \sum_{i=0}^{n}\left(\begin{array}{c}
n \\
i
\end{array}\right) D\left(A^{n-i} P^{i}\right)=\left(\sum_{i=0}^{n-1}\left(\begin{array}{c}
n-1 \\
i
\end{array}\right) A^{n-1-i} P^{i}\right) D(A+P)+ \\
& \left(\sum_{i=0}^{n-2}\left(\begin{array}{c}
n-2 \\
i
\end{array}\right) A^{n-2-i} P^{i}\right) D(A+P)(A+P)+ \\
& \left(\sum_{i=0}^{n-3}\left(\begin{array}{c}
n-3 \\
i
\end{array}\right) A^{n-3-i} P^{i}\right) D(A+P)(A+P)^{2}+\cdots+ \\
& (A+P)^{2} D(A+P)\left(\sum_{i=0}^{n-3}\left(\begin{array}{c}
n-3 \\
i
\end{array}\right) A^{n-3-i} P^{i}\right)+ \\
& (A+P) D(A+P)\left(\sum_{i=0}^{n-2}\left(\begin{array}{c}
n-2 \\
i
\end{array}\right) A^{n-2-i} P^{i}\right)+D(A+P)\left(\sum_{i=0}^{n-1}\left(\begin{array}{c}
n-1 \\
i
\end{array}\right) A^{n-1-i} P^{i}\right) \text {. }
\end{aligned}
$$

Using (1) and rearranging the equation (4) in sense of collecting together terms involving equal number of factors of $P$ we obtain: 


$$
\sum_{i=1}^{n-1} f_{i}(A, P)=0
$$

where $f_{i}(A, P)$ stands for the expression of terms involving $i$ factors of $P$.

Replacing $A$ by $A+2 P, A+3 P, \ldots, A+(n-1) P$ in turn in the equation (1), and expressing the resulting system of $n-1$ homogeneous equations of variables $f_{i}(A, P), i=1,2, \ldots, n-1$, we see that the coefficient matrix of the system is a van der Monde matrix

$$
\left[\begin{array}{cccc}
1 & 1 & \cdots & 1 \\
2 & 2^{2} & \cdots & 2^{n-1} \\
\vdots & \vdots & \vdots & \vdots \\
n-1 & (n-1)^{2} & \cdots & (n-1)^{n-1}
\end{array}\right] .
$$

Since the determinant of the matrix is different from zero, it follows that the system has only a trivial solution.

In particular,

$$
\begin{gathered}
f_{n-2}(A, P)=n(n-1) D\left(A^{2}\right)-(n-1)(n-2)\left(A^{2} D(P)+D(P) A^{2}\right)- \\
((n-2)(n-3)+(n-3)(n-4)+\cdots+3 \cdot 2+2 \cdot 1)\left(A^{2} D(P) P+P D(P) A^{2}\right)- \\
2((n-2)+(n-3)+(n-4)+\cdots+3+2+1)(A D(A) P+P D(A) A)- \\
4(1 \cdot(n-2)+2 \cdot(n-3)+3 \cdot(n-4)+\cdots+(n-3) \cdot 2+(n-2) \cdot 1) A D(P) A- \\
2(n-1)(A D(A)+D(A) A)=0,
\end{gathered}
$$

and

$$
\begin{gathered}
f_{n-1}(A, P)=n D(A)-(P D(A)+D(A) P)-(n-1)(A D(P)+D(P) A)- \\
((n-2)+(n-3)+(n-4)+\cdots+2+1)(A D(P) P+P D(P) A)- \\
(n-2) P D(A) P=0 .
\end{gathered}
$$

The above equations reduce to

$$
\begin{gathered}
n(n-1) D\left(A^{2}\right)=(n-1)(n-2)\left(A^{2} D(P)+D(P) A^{2}\right)+ \\
\frac{1}{3}(n-3)(n-2)(n-1)\left(A^{2} D(P) P+P D(P) A^{2}\right)+ \\
(n-2)(n-1)(A D(A) P+P D(A) A)+ \\
4(1 \cdot(n-2)+2 \cdot(n-3)+3 \cdot(n-4)+\cdots+(n-3) \cdot 2+(n-2) \cdot 1) A D(P) A+ \\
2(n-1)(A D(A)+D(A) A),
\end{gathered}
$$


and

$$
\begin{gathered}
2 n D(A)=2(P D(A)+D(A) P)+2(n-1)(A D(P)+D(P) A)+ \\
(n-2)(n-1)(A D(P) P+P D(P) A)+2(n-2) P D(A) P,
\end{gathered}
$$

respectively. Multiplying the relation (3) from both sides by $A$ we obtain

$$
A D(P) A=0,
$$

which reduces the relation (5) to

$$
\begin{gathered}
n(n-1) D\left(A^{2}\right)=(n-1)(n-2)\left(A^{2} D(P)+D(P) A^{2}\right)+ \\
\frac{1}{3}(n-3)(n-2)(n-1)\left(A^{2} D(P) P+P D(P) A^{2}\right)+ \\
(n-2)(n-1)(A D(A) P+P D(A) A)+ \\
2(n-1)(A D(A)+D(A) A) .
\end{gathered}
$$

Applying the relation (3) and the fact that $A P=P A=A$, we have $P D(P) A=(P D(P) P) A=$ 0 . Similarly one obtains that $A D(P) P=0$. The relations (8) and (6) can now be written as

$$
\begin{gathered}
n D\left(A^{2}\right)=(n-2)\left(A^{2} D(P)+D(P) A^{2}\right)+(n-2)(A D(A) P+P D(A) A)+ \\
2(A D(A)+D(A) A),
\end{gathered}
$$

and

$$
n D(A)=P D(A)+D(A) P+(n-1)(A D(P)+D(P) A)+(n-2) P D(A) P=0,
$$

respectively. Right multiplication of the relation (10) by $P$ gives

$$
D(A) P=D(P) A+P D(A) P .
$$

Similarly one obtains

$$
P D(A)=A D(P)+P D(A) P .
$$

Multiplying the relation (11) from the right side and the relation (12) from the left side by $A$, we obtain

$$
D(A) A=D(P) A^{2}+P D(A) A
$$

and

$$
A D(A)=A^{2} D(P)+A D(A) P
$$

Combining relations (9), (13) and (14) we obtain

$$
\begin{aligned}
& n D\left(A^{2}\right)=(n-2)\left(D(P) A^{2}+P D(A) A\right)+(n-2)\left(A^{2} D(P)+A D(A) P\right)+ \\
& 2(A D(A)+D(A) A)=(n-2)(A D(A)+D(A) A)+2(A D(A)+D(A) A) .
\end{aligned}
$$


We have therefore

$$
D\left(A^{2}\right)=D(A) A+A D(A)
$$

for any $A \in F(X)$. From the relation (10) one can conclude that $D(A) \in F(X)$ for any $A \in F(X)$. We have therefore a Jordan derivation on $F(X)$. Since $F(X)$ is prime it follows that $D$ is a derivation by Herstein's theorem. Applying Theorem A one can conclude that $D$ is of the form

$$
D(A)=A B-B A,
$$

for all $A \in A(X)$ and some $B \in L(X)$. It remains to prove that the relation (16) holds on $A(X)$ as well. Let us introduce $D_{1}: A(X) \rightarrow L(X)$ by $D_{1}(A)=A B-B A$ and consider $D_{0}=D-D_{1}$. The mapping $D_{0}$ is, obviously, linear and satisfies the relation (1). Besides, $D_{0}$ vanishes on $F(X)$. It is our aim to prove that $D_{0}$ vanishes on $A(X)$ as well. Let $A \in A(X)$, let $P$ be an one-dimensional projection and $S=A+P A P-(A P+P A)$. We have $D_{0}(S)=D_{0}(A)$. and $S P=P S=0$. We have

$$
D_{0}\left(A^{n}\right)=\sum_{j=1}^{n} A^{n-j} D_{0}(A) A^{j-1}
$$

for all $A \in A(X)$. Applying the above relation we obtain

$$
\begin{gathered}
\sum_{j=1}^{n} S^{n-j} D_{0}(S) S^{j-1}=D_{0}\left(S^{n}\right)=D_{0}\left(S^{n}+P\right)=D_{0}\left((S+P)^{n}\right)= \\
\sum_{j=1}^{n}(S+P)^{n-j} D_{0}(S+P)(S+P)^{j-1}=\sum_{j=1}^{n}(S+P)^{n-j} D_{0}(A)(S+P)^{j-1}= \\
\sum_{j=1}^{n}\left(S^{n-j}+P\right) D_{0}(S)\left(S^{j-1}+P\right)=\sum_{j=1}^{n} S^{n-j} D_{0}(A) S^{j-1}+ \\
\sum_{j=1}^{n} P D_{0}(A) S^{j-1}+\sum_{j=1}^{n} S^{n-j} D_{0}(A) P+P D_{0}(A) P .
\end{gathered}
$$

We have therefore

$$
\sum_{j=1}^{n} P D_{0}(A) S^{j-1}+\sum_{j=1}^{n} S^{n-j} D_{0}(A) P+P D_{0}(A) P=0
$$

Multiplying the above relation from both sides by $P$ we obtain

$$
P D_{0}(A) P=0
$$

which reduces the relation (18) to

$$
\sum_{j=1}^{n} P D_{0}(A) S^{j-1}+\sum_{j=1}^{n} S^{n-j} D_{0}(A) P=0 .
$$


Right multiplication of the above relation by $P$ gives

$$
\sum_{j=1}^{n} S^{n-j} D_{0}(A) P=0 .
$$

Let us prove that

$$
\sum_{j=1}^{n-1} k_{j} S^{n-1-j} D_{0}(A) P=0
$$

holds where $k_{j}=2^{n-1-j}-2^{n-1}, j=1,2, \ldots, n-1$. Putting in the relation (21) $2 A$ for $A$ we obtain

$$
\sum_{j=1}^{n} 2^{n-j} S^{n-j} D_{0}(A) P=0
$$

Multiplying the relation (21) by $2^{n-1}$ and subtracting the relation so obtained from the above relation we obtain the relation (22). Since the relation (21) implies the relation (22) one can conclude by induction that $D_{0}(A) P=0$. Since $P$ is an arbitrary one-dimensional projection, it follows that $D_{0}(A)=0$, for any $A \in A(X)$, which completes the proof of the theorem.

Let us point out that in case $n=3$ Theorem 1 reduces to Theorem in [9].

THEOREM 2. Let $A$ be a semisimple $H^{*}$-algebra and let $D: R \rightarrow R$ be a linear mapping satisfying the relation

$$
D\left(x^{n}\right)=\sum_{j=1}^{n} x^{n-j} D(x) x^{j-1}
$$

for all $x \in R$. In this case $D$ is a linear derivation.

Proof. The proof goes through using the same arguments as in the proof of Theorem in [5] with the exception that one has to use Theorem 1 instead of Lemma in [5].

Since in the formulation of the results presented in this paper we have used only algebraic concepts, it would be interesting to study the problem in a purely ring theoretical context. We conclude with the following conjecture.

CONJECTURE. Let $R$ be a semiprime ring with suitable torsion restrictions and let $D: R \rightarrow R$ be an additive mapping satisfying the relation

$$
D\left(x^{n}\right)=\sum_{j=1}^{n} x^{n-j} D(x) x^{j-1}
$$

for all $x \in R$. In this case $D$ is a derivation.

In case $R$ has the identity element the conjecture above was proved in [8]. Since semisimple $H^{*}$-algebras are semiprime, Theorem 2 proves the conjecture above in a special case.

$$
\text { Received: June, 2008. Revised: October, } 2009 .
$$




\section{References}

[1] Ambrose, W., Structure theorems for a special class of Banach algebras, Trans. Amer. Math. Soc., 57 (1945), 364-386.

[2] Chernoff, P.R., Representations, automorphisms, and derivations of some operator algebras, J. Funct. Anal., 2 (1973), 275-289.

[3] Cusack, J., Jordan derivations on rings, Proc. Amer. Math. Soc., 53 (1975), 321-324.

[4] Herstein, I.N., Jordan derivations of prime rings, Proc. Amer. Math. Soc., 8 (1957), 11041119.

[5] Molnár, L., On centralizers of an $H^{*}$-algebra, Publ. Math. Debrecen, 46, 1-2 (1995), 89-95.

[6] Šemrl, P., Ring derivations on standard operator algebras, J. Funct. Anal., 112 (1993), $318-324$.

[7] Vukman, J., On automorphisms and derivations of operator algebras, Glasnik Mat., 19 (1984), 135-138.

[8] Vukman, J. And Kosi-Ulbl, I., A note on derivations in semiprime rings, Internat. J. Math. \&Math. Sci., 20 (2005), 3347-3350.

[9] Vukman, J., On derivations of standard operator algebras and semisimple $H^{*}$-algebras, Studia Sci. Math. Hungar., 44 (2007), 57-63. 